\title{
Diversity and Identification of Yeasts Isolated from Tumultuous Stage of Spontaneous Table Grape Fermentations in Central China
}

\author{
Ye D.-Q. ${ }^{1}$, Sun Y. ${ }^{2}$, Song Y.-Y. ${ }^{1}$, Ma X.-L. ${ }^{1}$, Ren X.-N. ${ }^{1}$, Gong X., ${ }^{1}$ Liu Y.-L. ${ }^{1 *}$ \\ (1) College of Enology, Northwest A\&F University, Yangling 712100, Shaanxi, China \\ (2) Wine School, Ningxia University, Yinchuan, Ningxia 750021, China \\ Ye D.-Q and Sun Y. contributed equally to the study. \\ Submitted for publication: May 2018 \\ Accepted for publication: August 2018
}

Keywords: Table grape, spontaneous fermentation, autochthonous yeast, interdelta analysis, S. cerevisiae

\begin{abstract}
Table grapes are of increasing interest for wine production in China. In this study, 480 yeast isolates were isolated from the tumultuous stage during the spontaneous fermentation of six table grape varieties, which were cultivated in an ecological environment that was not industry-influenced, in Central China. The 26S rDNA D1/D2 domain sequence analysis was more efficient for yeast species identification than the 5.8S-ITS region RFLP analysis in the present study. All the tested strains belonged to nine species from six genera: Hanseniaspora guilliermondii, $H$. opuntiae, H. uvarum, Pichia terricola, Kazachstania hellenica, K. zonata, P. occidentalis, Saccharomyces cerevisiae and Zygosaccharomyces bailii. The yeast species and populations differed notably among the grape varieties. $S$. cerevisiae was found in the samples of four grape varieties (Vitis amurensis, Iona, Moldova and $V$. davidii), but not detected in Cuihong and Alimandeng Rose. Interdelta sequence fingerprinting analysis was used to discriminate between 128 . cerevisiae isolates. Eight $S$. cerevisiae genotypes (G1 to G8) were distinguished. Genotypes G1, G2, and G3 were the most dominant strains, accounting for $32.03 \%, 24.22 \%$ and $28.13 \%$ of the isolates respectively. This study shows the diversity of yeast species associated with spontaneous fermentations of different table grape varieties grown in an ecological environment without any wine industry effect or footprint.
\end{abstract}

\section{INTRODUCTION}

Table grapes are ranked one of the most economically important fruit in China, and made up $83 \%$ of total grape production in 2015 (OIV, 2017). As the world's largest table grape producer, China contributes over one-third of global productivity (FAO \& OIV, 2016). However, there are some problems behind the rapid increase in table grape production; one is that there was an obvious production-consumption surplus in some regions (Feng et al., 2014). To further increase the value, the interest in the production of wine using table grapes has been growing, especially since there is an oversupply of table grapes in the market. Yeasts are mainly responsible for the alcoholic fermentation of grape juice, which can be a sequential development of various genera, species and strains (Valero et al., 2007; Lopandic et al., 2008; Sun et al., 2014). Generally, non-Saccharomyces yeasts such as Hanseniaspora/Kloeckera, Metschnikowia, Candida, Pichia and Kluveromyces are present in the early stages of grape must fermentation (Fleet, 1998). As fermentation progresses, Saccharomyces cerevisiae dominates the middle until the end stage of wine fermentation, with an increase in ethanol concentration (Bisson \& Kunkee, 1993; Goddard \& Greig, 2015). Non-Saccharomyces yeasts also participate in the fermentation process (Torija et al., 2001; Di Maro et al., 2007; Zhang et al., 2010). The diversity of yeast communities present on grapes and in must is affected by climatic conditions, age of the vineyards, grape variety, viticulture and oenological practices (Renouf et al., 2007; Mercado et al., 2010; Drumonde-Neves et al., 2017).

While several studies have focused on monitoring indigenous yeasts during spontaneous wine grape fermentations in several viticulture regions in China (Sun et al., 2009; Li et al., 2011; Wang \& Liu 2013; Sun \& Liu 2014; Sun et al., 2014), such studies have not been published about table grapes. The red grapes Vitis amurensis, Cuihong, Alimandeng Rose, Moldova and Vitis davidii, and the white grape Iona are table grape cultivars grown at the Zhengzhou Fruit Research Institute (ZFRI) in the central region of China. ZFRI is an important grapevine preservation institute in China and contains abundant germplasm resources of grapes (Jiang et al., 2015). It experiences a monsoon-

\footnotetext{
*Corresponding author: E-mail address: yanlinliu@nwsuaf.edu.cn [Tel./Fax: 0086-29-87092931]

Acknowledgments: This study was supported by the National Natural Science Fund Programme (31571812, 31501463), the China Agriculture Research System (CARS-29-jg-03), the open funds of the Key Laboratory of Viticulture and Oenology, Ministry of Agriculture, China (KLVE201702), and the Adaptability, demonstration and promotion of wine grape varieties in the Northern piedmont of Tianshan Montation in Xinjiang (201604D132034). We are grateful to Conghuai Liu, Sanjun Liu and Jingnan Guo, from ZFRI, the Chinese Academy of Agriculture Sciences, for providing the grape samples and assistance
} 
influenced, four-season humid subtropical climate, with cool, dry winters and hot, humid summers. Table grapes are intended for consumption and those used in winemaking are different from wine grapes. Although they are mainly used as table grapes for consumption, many local growers also use them for wine fermentation, obtaining better value from wines, especially when table grapes are in excess supply and the price drops substantially. Although some work has been done on the table grape microbiome, most researchers have focused on the surface of the berry during the mature stage, and concentrated on the control of postharvest diseases (Kántor \& Kačániová, 2015; Carmichael et al., 2017). Little has been done to study yeast species involved in table grape fermentations. No winery has been established in this region, indicating that the chance of commercial yeast occurring is scarce. In other words, it is good to investigate the yeast biodiversity in those vineyards without a wine industry influence.

The objective of this study was to investigate the yeast populations of spontaneous table grape fermentations and their possible correlation with grape varieties grown in an ecological environment where there is no wine industry effect. In this study, autochthonous yeasts were isolated from the fermenting must of six different table grape varieties grown in ZFRI. Information generated from this study is of value for the development of table grapes for wine production.

\section{MATERIALS AND METHODS \\ Yeast isolation}

Vitis amurensis (Rupr.), Iona, Cuihong, Alimandeng Rose, Moldova, and $V$. davidii were grown in the same ecological environment in the vineyard of ZFRI (113 $\left.42^{\prime} \mathrm{E}, 34^{\circ} 43^{\prime} \mathrm{N}\right)$. The grape samples were collected from each variety in duplicate in 2012. No commercial dry yeast for wine fermentation has ever been used in this region. Approximately $2 \mathrm{~kg}$ of grapes with stems were harvested in aseptic conditions from each sampling point and placed directly into sterile bags, which were transported to the laboratory in portable refrigerators with ice bricks and processed within $1 \mathrm{~h}$. The initial sugar concentrations ranged between 120 and
$210 \mathrm{~g} / \mathrm{L}$, and the physicochemical parameters were listed in Table 1. Each grape sample was hand squeezed directly into sterile bags using a different pair of sterile gloves for each sample, and then the grape must was aseptically transferred into sterile flasks $(1.5 \mathrm{~L})$. The bottles were covered with sterile two-layer gauze to avoid contamination (ClementeJimenez et al., 2004; Sun \& Liu, 2014), and the grape must fermentation was allowed to proceed spontaneously at $24^{\circ} \mathrm{C}$ to $26^{\circ} \mathrm{C}$ for each grape variety in duplicate. Fermentations were monitored for weight loss (as gram of $\mathrm{CO}_{2}$ ) per 12 hours (Fig. 1). Wines were sampled during the tumultuous stage (sugar consumption was about 2/3, as shown in Fig. 1) of the fermentation. These samples were serially diluted before plating out on YPD agar $(10 \mathrm{~g} / \mathrm{L}$ yeast extract, $20 \mathrm{~g} / \mathrm{L}$ peptone, $20 \mathrm{~g} / \mathrm{L}$ glucose and $20 \mathrm{~g} / \mathrm{L}$ agar, natural $\mathrm{pH}$ ), which was supplemented with $100 \mathrm{mg} / \mathrm{L}$ chloramphenicol to inhibit bacterial growth. Triplicate samples were plated out. The plates were inoculated at $28^{\circ} \mathrm{C}$ for two to three days, and 10 to 15 colonies from each plate were randomly selected ( 80 colonies/variety) and preserved at $-20^{\circ} \mathrm{C}$ in $20 \%$ (v/v) glycerol. Thus, the 480 pure cultures selected were differentiated and classified according to colony morphology and colour on Wallerstein nutrient (WLN) agar (Pallmann et al., 2001) and grown for five days at $28^{\circ} \mathrm{C}$.

\section{Identification of yeast culture}

Yeast DNA was extracted according to the method of Sun and Liu (2014). Five to eight representative colonies of each phenotype on WLN agar were identified by sequence analysis of the 26S rDNA D1/D2 domain and 5.8S-ITS-RFLP. The 26S rDNA D1/D2 domain was amplified using primers NL1 and NL4 according to Kurtzman and Robnett (1998). The PCR procedure and the subsequent visualisation steps were done as described as Wang and Liu (2013). The products that gave positive results were sent to Beijing Sunbiotech Co. Ltd. for purification and sequence determination. The sequences were analysed using the Blast method of NCBI (http://www. ncbi.nlm.nih.gov/blast). The 5.8S-ITS rDNA was amplified by PCR using primers ITS1 and ITS4, as described by White et al. (1990). The PCR procedure and subsequent visualisation steps were carried out as described by Sun and

TABLE 1

Physicochemical parameters of the must and wine in different fermentations.

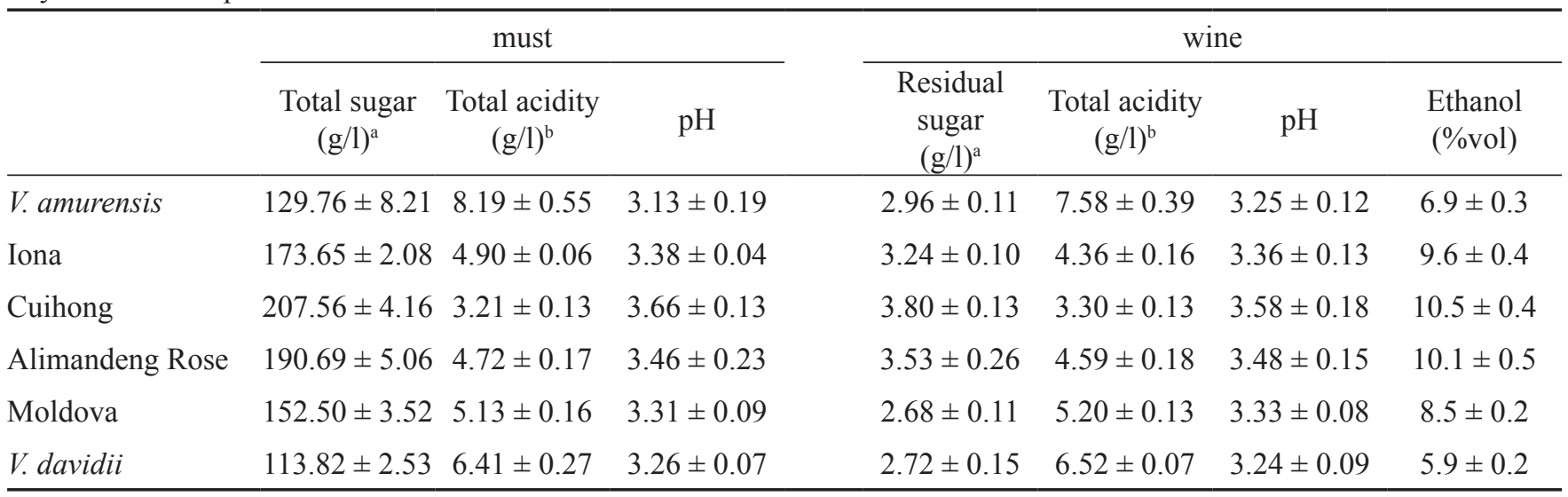

${ }^{a}$ Concentration represented by glucose

${ }^{\mathrm{b}}$ Concentration represented by tartaric acid 

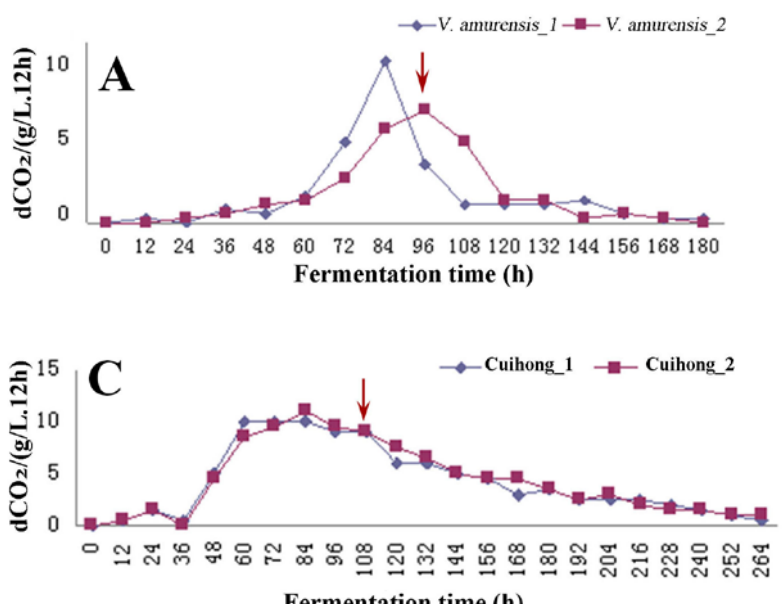

Fermentation time (h)

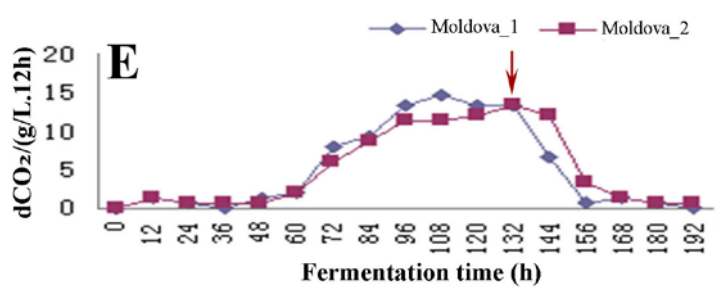

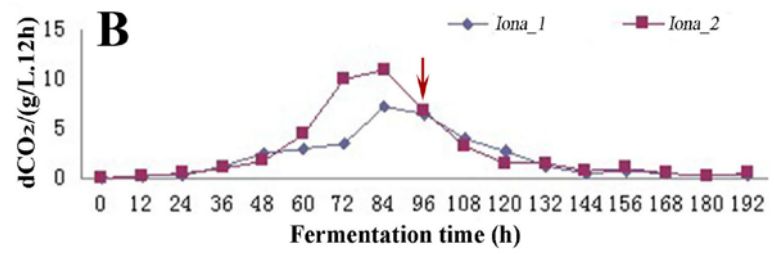
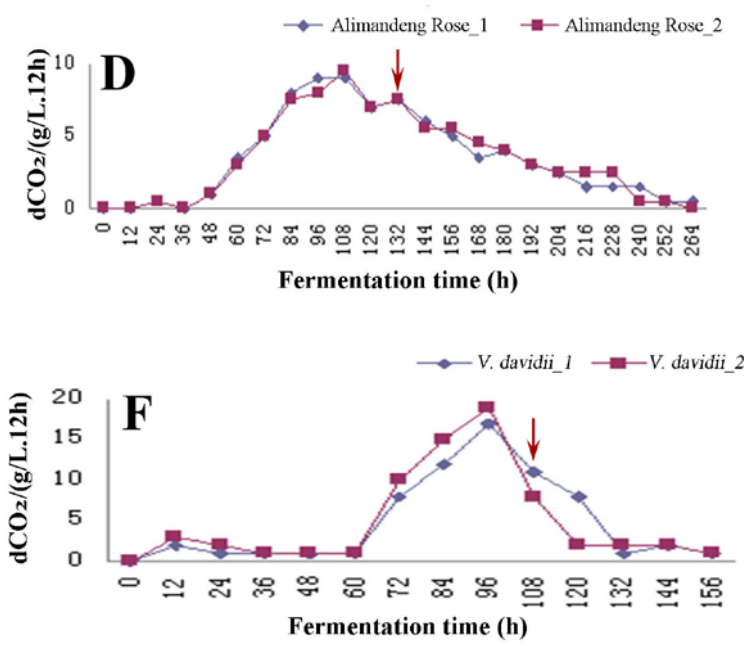

FIGURE 1

The spontaneous fermentation curves of six table grape varieties. The red arrow indicates the sampling point

Liu (2014). The PCR products were digested separately with two different restriction endonucleases, HaeIII and Hinfl. The digestions were performed according to the instructions of the supplier, TaKaRa Biotechnology (Dalian) Co. Ltd. The size of the restriction fragments was obtained by separation on $3 \%(\mathrm{w} / \mathrm{v})$ agarose gels and comparing them to the 2000 DNA Marker (TaKaRa).

\section{Interdelta sequence typing}

Interdelta sequence typing of all the $S$. cerevisiae isolates was carried out as described by Sun et al. (2014), using delta12 and delta2 1 primers according to Legras and Karst (2003).

\section{RESULTS AND DISCUSSION}

\section{Identification of yeast isolates}

Yeast were isolated and identified from the spontaneous fermentations of six table grape cultivars, namely $V$. amurensis, Iona, Cuihong, Alimandeng Rose, Moldova and $V$. davidii. As shown in Table 2, nine species (groups), viz. H. guilliermondii, $H$. opuntiae, $H$. uvarum, P. terricola, Kazachstania hellenica, K. zonata, Pichia occidentalis, S. cerevisiae and Zygosaccharomyces bailii were identified by sequencing of the $26 \mathrm{~S}$ rDNAD1/D2 region. In addition, the PCR products of 5.8S-ITS rDNA were digested with HaeIII and Hinfl, whereby eight different profiles were obtained. Seven of the nine species were identified after comparing the molecular weight of the restriction products digested by restriction endonuclease HaeIII and Hinfl with those previously described by Esteve-Zarzoso et al. (1999) and the Yeast-id database (http://yeast-id.com/). H. guilliermondii (Group I), H. uvarum (Groups II and III), P. terricola
(Group IV), T. delbrueckii (Group V), S. cerevisiae (Group VIII), and Z. bailii (Group IX). Group VII was identified as C. sorbosa/P. terricola/P. galeiformis, while Group VI showed no match to any species in the database.

For H. guilliermondii (Group I), P. terricola (Group IV), S. cerevisiae (Group VIII) and Z. bailii (Group IX), the two methods coincided with regard to yeast species assignment. Similar to the findings of Wang and Liu (2013), H. opuntiae (Group II) and H. uvarum (Group III) shared similar 5.8S-ITS rDNA restriction patterns. However, this study showed that the 26S rDNA D1/D2 domain sequence of these two species differed. Isolates from group VI could not be identified with 5.8S-ITS rDNA-RFLP analysis, but were identified as $K$. zonata with the $26 \mathrm{~S}$ rDNA D1/D2 sequence analysis. For isolates from groups V and VII, the 26S rDNA D1/D2 sequences were used for species identification, because no information about 5.8S-ITS rDNA restriction patterns for $K$. hellenica and $P$. occidentalis was available. Therefore, we recommend that the $26 \mathrm{~S}$ rDNA D1/D2 sequence analysis should be the first choice for yeast species identification. It is not necessary to perform 5.8S-ITS rDNA-RFLP analysis, as the estimation of the sizes of fragments may vary using electrophoresis in different laboratories, and the 5.8S-ITS rDNA RFLP profile database may not cover all known yeast species.

\section{Population diversity of yeasts from different table grape varieties}

Generally, three to five different yeast species were isolated from wines produced from each of the various grape varieties (Fig. 2). The yeast species and the corresponding populations 


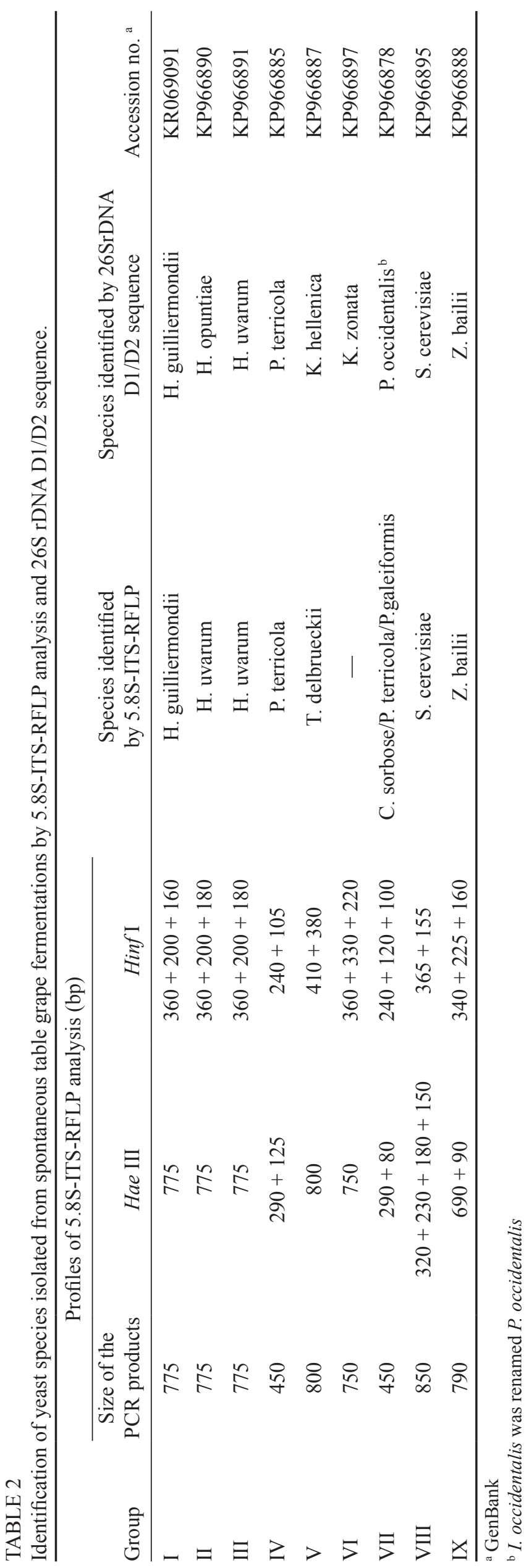

exhibited differences among the grape varieties. $K$. hellenica $(51.25 \%)$ was dominant in $V$. amurensis fermentations, and its high numbers could be a result of the humid summers in Zhengzhou. A study by Nisiotou and Nychas (2008) showed the linkage of Kazachstania sp. with Botrytis-affected fermenting grape juice. S. cerevisiae was predominant in the Iona and Moldova fermentations (53.75\% and $96.25 \%$ respectively), while $H$. uvarum was predominant in the Cuihong, Alimandeng Rose and $V$. davidii fermentations $(62.50 \%, 58.75 \%$ and $66.25 \%$ respectively). These findings are in agreement with other studies (Di Maro et al., 2007; Zhang et al., 2010; Li et al., 2011; Sun et al., 2014). It is widely reported that $H$. uvarum is one of the most frequently encountered species in wine fermentations, and it shows positive oenological properties for wine production ( $\mathrm{Li}$ et al., 2011). Other minor non-Saccharomyces species, such as $H$. opuntiae, were isolated in the fermentations of three grape varieties (Cuihong, Alimandeng Rose and Moldova). P. terricola and P. occidentalis were isolated from four grape varieties (Vitis amurensis, Iona, Cuihong and $V$. davidii). Species of $H$. guilliermondii, $K$. zonata, and $Z$. bailii occurred at extremely low populations in the fermentation of Iona, Cuihong and Alimandeng Rose, and only one colony among the 80 random yeast isolates was obtained from the corresponding samples. This association could be attributed to differences in the physicochemical properties of the different grape varieties, which affect the adaptation of wild yeasts (Rojas et al., 2003).

Although the biodiversity of wine yeasts has been analysed in different viticultural regions in China (Li et al., 2011; Wang \& Liu, 2013; Sun et al., 2014), this is the first study conducted on autochthonous yeast biodiversity in table grape vineyards that have never been exposed to commercial wine yeasts. This result contributes to the understanding of yeast biodiversity within and between vineyards located in the same ecological environment, as described by Mercado et al. (2010).

\section{S. cerevisiae diversity determined by interdelta sequence typing}

$S$. cerevisiae strain diversity with regard to the four grape varieties is listed in Table 3. The banding patterns that were generate for the 128 isolates showed eight different genotypes, named as G1 to G8 (Fig. 3). Among these S. cerevisiae isolates, predominant genotypes were G1, G2 and G3, with proportions of $32.03 \%, 24.22 \%$ and $28.13 \%$ respectively (Table 3). G4 accounted for around $10 \%$ of the total isolates, and the other three genotypes occurred rarely (below 2\%). The degree of variability, measured as the percentage of different strains found amongst the colonies analysed (Torija et al., 2001), was 6.25\%. Moreover, the distribution of $S$. cerevisiae genotypes differed among the grape varieties. $V$. amurensis and Iona shared the same predominant genotype (G3), with a proportion of $81.82 \%$ and $62.79 \%$ respectively. $S$. cerevisiae isolates with the G1 genotype dominated as a proportion of $73.08 \%$ in $\mathrm{V}$. davidii fermentations, while in Moldova fermentations, $47.92 \%$ of S. cerevisiae isolates had the G2 genotype.

It has been reported that fermentative species of Saccharomyces (S. cerevisiae) occur at extremely low 
populations on intact berries, and that these species originate from the equipment surfaces in the winery during spontaneous fermentations, and even from insect vectors such as bees and wasps (Martini, 1993; Mortimer \& Polsinelli, 1999). Since there is no winery in Zhengzhou, the possibility of commercial yeast colonisation in ZFRI should be very low, indicating that the $S$. cerevisiae strains isolated in this study came from the vineyard. G1, G2, G4, G6, G7 and G8 appear to be new genotypes when compared to the previous studies of Pei et al. (2009) and Sun et al. (2015). The G3 genotype is similar to genotype I found by Sun et al. (2015), while G5 is similar to genotype V found by Pei et al. (2009). Studies by Tofalo et al. $(2013,2014)$ reveal that unique $S$. cerevisiae strains are associated with particular grape varieties in specific geographical locations. Characterising the yeast community and its oenological properties in a specific wine could provide data for potential industrial applications ( $\mathrm{Li}$ et al., 2011; Tofalo et al., 2014).

Eight different $S$. cerevisiae genotypes revealed considerable strain diversity among the table grapes fermentations. Moreover, differences in genotype distribution were found according to grape varieties, which is in

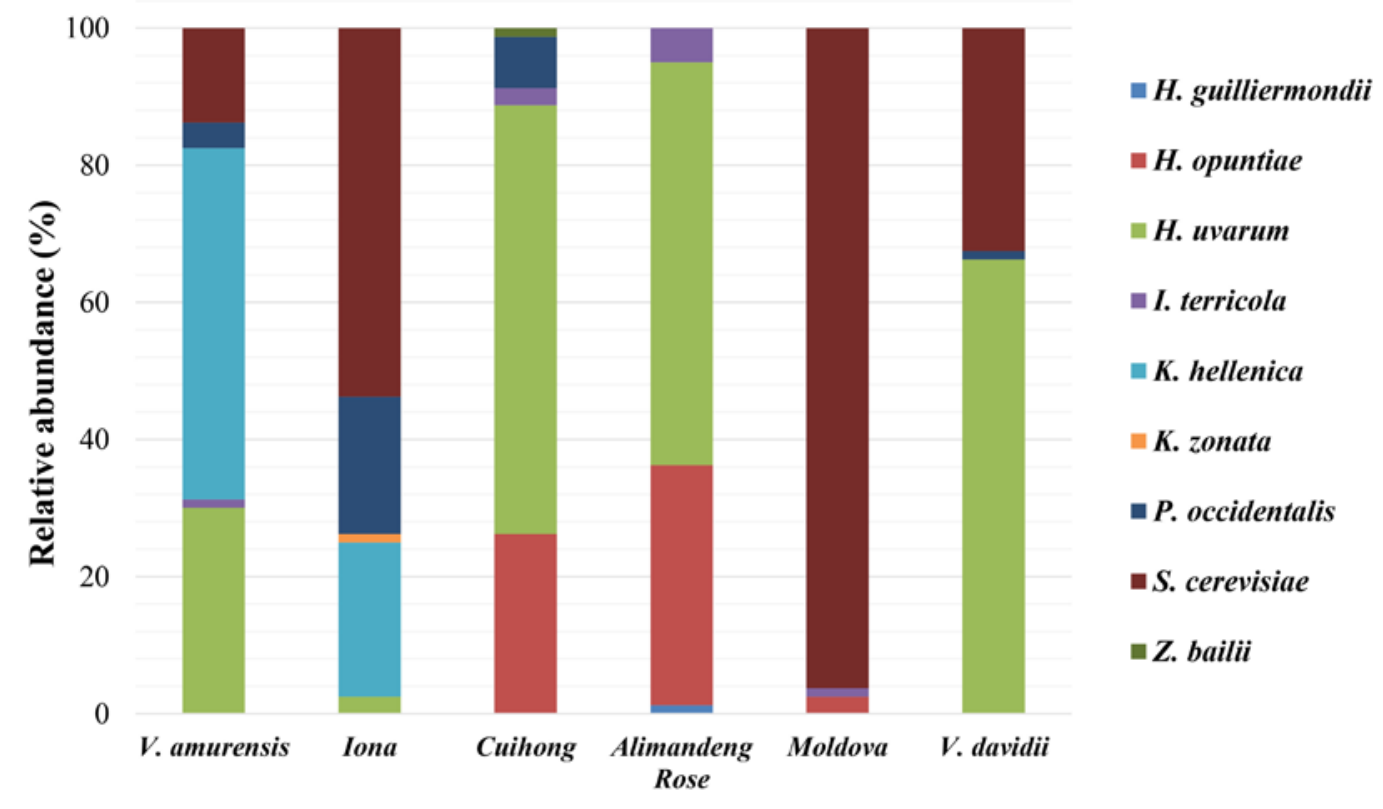

Grape varieties

FIGURE 2

The occurrence of yeast species at the tumultuous stages in spontaneous fermentations for different table grape varieties

TABLE 3

Distribution and proportion of $S$. cerevisiae genotypes of different table grape varieties.

\begin{tabular}{|c|c|c|c|c|c|}
\hline \multirow[b]{2}{*}{ Genotypes } & \multicolumn{4}{|c|}{ Numbers of each genotype/proportion (\%) ${ }^{a}$} & \multirow[b]{2}{*}{ Total $/(\%)^{b}$} \\
\hline & V. amurensis & Iona & Moldova & V. davidii & \\
\hline G1 & - & - & $22 / 45.83$ & $19 / 73.08$ & $41 / 32.03$ \\
\hline $\mathrm{G} 2$ & $1 / 9.09$ & - & $23 / 47.92$ & $7 / 26.92$ & $31 / 24.22$ \\
\hline G3 & $9 / 81.82$ & $27 / 62.79$ & - & - & $36 / 28.13$ \\
\hline G4 & $1 / 9.09$ & $13 / 30.23$ & - & - & $14 / 10.94$ \\
\hline G5 & - & $2 / 4.65$ & - & - & $2 / 1.56$ \\
\hline G6 & - & $1 / 2.33$ & - & - & $1 / 0.78$ \\
\hline G7 & - & - & $1 / 2.08$ & - & $1 / 0.78$ \\
\hline G8 & - & - & $2 / 4.17$ & - & $2 / 1.56$ \\
\hline
\end{tabular}

${ }^{a}$ Percentage calculated from each variety

${ }^{\mathrm{b}}$ Percentage calculated from the total 128 isolates

-: undetected 


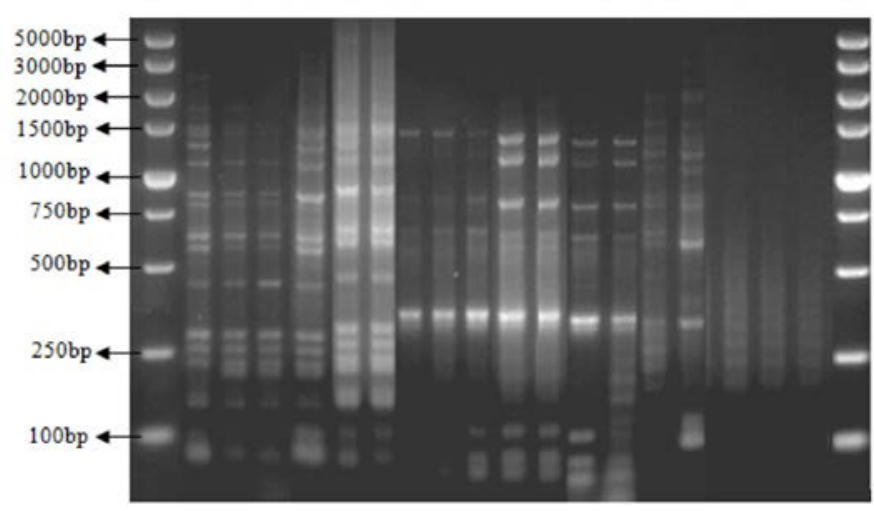

FIGURE 3

The interdelta sequence profiles of the 128 Saccharomyces cerevisiae isolates studied. M: 5000 bp DNA Ladder; G1 to G8 represent different genotypes. Roman numbers VI, VII, IX indicate the non-Saccharomyces species K. zonata, P. occidentalis and $Z$. bailii respectively.

agreement with previous studies showing that the presence or absence of $S$. cerevisiae differed according to each plant and grape cluster (Pretorius et al., 1999). It is well known that, in most cases, $S$. cerevisiae is the main species occurring in the tumultuous stages of fermentation, and that it prevails until the fermentation has finished. However, in this study, twothirds of the samples of table grapes were not predominantly fermented by $S$. cerevisiae. $S$. cerevisiae was not found at the tumultuous stages in the fermentations of Cuihong and Alimandeng Rose. Drumonde-Neves et al. (2017) reported similar results, namely that $25 \%$ of fermentations were finished by non-Saccharomyces species on five islands of the Azores Archipelago, Spain. The reasons why S. cerevisiae was not found were not clear, but one possible explanation is that its presence and diversity during wine fermentations are complex and unpredictable (Sun et al., 2015). Many factors, such as climatic conditions, age of the vineyards, grape varieties and viticulture, can affect the diversity of yeasts during wine fermentation (Mortimer \& Polsinelli, 1999; Pretorius et al., 1999; Clemente-Jimenez et al., 2004; Drumonde-Neves et al., 2017). Consequently, to obtain more reliable results, other information, such as the nitrogen concentration of the grapes as well as the composition of the microbial community in the phyllosphere, should be known in order to do further analysis (Oliveira et al., 2017).

\section{CONCLUSIONS}

Table grapes are of increasing interest for wine production in China. However, little has been done to study the yeast species involved in table grape fermentations. The present study has shown the yeast biodiversity involved in the spontaneous fermentations of different table grape varieties. Differences between cultivars were apparent, as two-thirds of the samples were predominantly fermented by non-Saccharomyces species in the tumultuous stages a distinct difference compared to wine grape fermentation. This would provide a starting point for future table grape wine ecology/alcoholic beverage research. Further research on the oenological properties of these yeast species could be valuable for the evaluation of wine quality during native fermentations.

\section{LITERATURE CITED}

Bisson, L.F. \& Kunkee, R.E., 1993. Microbial interactions during wine production. In: Zeikus, J.G. \& Johnson, E.A. (eds). Mixed cultures in biotechnology. McGraw-Hill, New York. pp. $37-68$.

Carmichael, P.C., Siyoum, N., Chidamba, L. \& Korsten, L., 2017. Characterisation of fungal communities of developmental stages in table grape grown in the northern region of South Africa. J. of Appl. Microbiol. 123(5), 1251-1262.

Clemente-Jimenez, J.M., Mingorance-Cazorla, L., Martínez-Rodríguez, S., Las Heras-Vázquez, F.J. \& Rodríguez-Vico, F., 2004. Molecular characterization and oenological properties of wine yeasts isolated during spontaneous fermentation of six varieties of grape must. Food Microbiol. $21,149-155$.

Di Maro, E., Ercolini, D. \& Coppola, S., 2007. Yeast dynamics during spontaneous wine fermentation of the Catalanesca grape. Int. J. Food Microbiol. 117, 201-210.

Drumonde-Neves, J., Franco-Duarte, R., Lima, T., Schuller, D. \& Pais, C., 2017. Association between grape yeast communities and the vineyard ecosystems. PLoS One, 12(1), e0169883.

Esteve-Zarzoso, B., Belloch, C., Uruburu, F. \& Querol, A., 1999. Identification of yeasts by RFLP analysis of the 5.8S rRNA gene and the two ribosomal internal transcribed spacers. Int. J. Syst. Bacteriol. 49, 329-337.

FAO \& OIV, 2016. Table and dried grapes. International Organisation of Vine and Wine. http://www.oiv.int/public/medias/5116/booklet-fao-oivgrapes-focus.pdf

Feng, J., Xia, W., Fu, Z. \& Mu, W., 2014. Assessment of consumers' perception and cognition toward table grape consumption in china. Brit. Food J. 116(4), 189-194.

Fleet, G.H., 1998. The microbiology of alcoholic beverages. In: Wood, B.J.B. (ed.). Microbiology of fermented foods. Blackie Academic \& Professional, New York. pp. $217-262$.

Goddard, M.R. \& Greig, D., 2015. Saccharomyces cerevisiae: A nomadic yeast with no niche? FEMS Yeast Res. 15, 1-6. 
Jiang, J.F., Kell, S., Fan, X.C., Zhang, Y., Wei, W., Kang, D.M., Maxted, N., Ford-Lloyd, B. \& Liu, CH., 2015. The wild relatives of grape in China: Diversity, conservation gaps and impact of climate change. Agric. Ecosyst. Environ. 210, 50-58.

Kántor, A. \& Kačániová, M. 2015. Diversity of bacteria and yeasts on the surface of table grapes. Sci Papers Anim. Sci. Biotechnol. 48(1), 149-155.

Kurtzman, C.P. \& Robnett, C.J., 1998. Identification and phylogeny of ascomycetous yeasts form analysis of nuclear large subunit 26S ribosomal DNA partial sequences. Antonie Leeuwenhoek 73, 337-371.

Legras, J.L. \& Karst, F., 2003. Optimisation of interdelta analysis for Saccharomyces cerevisiae strain characterization. FEMS Microbiol. Lett. $221,249-255$

Li, E.H., Liu, A.G., Xue, B. \& Liu, Y.L., 2011. Yeast species associated with spontaneous wine fermentation of Cabernet Sauvignon from Ningxia, China. World J. Microbiol. Biotechnol. 27, 2475-2482.

Lopandic, K., Tiefenbrunner, W., Gangl, H., Mandl, K., Berger, S., Leitner G., Abd-Ellah, G.A., Querol, A., Gardner, R.C., Sterflinger, K. \& Prillinger, H., 2008. Molecular profiling of yeasts isolated during spontaneous fermentations of Austrian wines. FEMS Yeast Res. 8, 1063-1075.

Martini, A., 1993. Origin and domestication of the wine yeast Saccharomyces cerevisiae. J. Wine Res. 4, 165-176.

Mercado, L., Jubany, S., Gaggero, C., Masuelli, R.W. \& Combina, M., 2010 Molecular relationships between Saccharomyces cerevisiae strains involved in winemaking from Mendoza, Argentina. Curr. Microbiol. 61, 506-514.

Mortimer, R. \& Polsinelli, M. 1999. On the origins of wine yeast. Res. in Microbiol. 150(3), 199-204.

Nisiotou, A.A. \& Nychas, G.E., 2008. Kazachstania hellenica sp. nov., a novel ascomycetous yeast from a Botrytis-affected grape must fermentation. Int. J. Syst. Bacteriol. 58, 1263-1267.

OIV, 2017. Statistical report on world vitiviniculture. OIV, Paris.

Oliveira, M., Arenas, M., Lage, O., Cunha, M. \& Amorim, M. I., 2017. Epiphytic fungal community in Vitis vinifera of the Portuguese wine regions. Lett. Appl. Microbiol, 66(1), 93-102.

Pallmann, C.L., Brown, J.A., Olineka, T.L., Cocolin, L., Mills, D.A. \& Bisson, L.F., 2001. Use of WL medium to profile native flora fermentations. Am. J. Enol. Vitic. 52, 198-203.

Pei, Y.F., Wang, G.P. \& Liu, Ye.L., 2009. Strain typing of Saccharomyces cerevisiae during spontaneous fermentation of Cabernet Sauvignon wine Microbiol. China 10, 1532-1535.

Pretorius, I.S., Van der Westhuizen, T.J. \& Augustyn, O.P.H., 1999. Yeast biodiversity in vineyards and wineries and its importance to the South African wine industry. S. Afr. J. Enol. Vitic. 20, 61-74.
Renouf, V., Claisse, O. \& Lonvaud-Funel, A., 2007. Inventory and monitoring of wine microbial consortia. Appl. Microbiol. Biotechnol. 75, 149-164.

Rojas, V., Gil, J., Piñaga, F. \& Manzanares, P., 2003. Acetate ester formation in wine by mixed cultures in laboratory fermentations. Int. J. Food Microbiol. 86, 181-188.

Sun, H.H., Ma, H.Q., Hao, M.L., Pretorius, I.S. \& Chen, S.W., 2009. Identification of yeast population dynamics of spontaneous fermentation in Beijing wine region, China Ann. Microbiol. 59, 69-76.

Sun, Y. \& Liu, Y.L., 2014. Investigating of yeast species in wine fermentation using terminal restriction fragment length polymorphism method. Food Microbiol. 38, 201-207.

Sun, Y., Guo, J.J., Liu, F.B. \& Liu, Y.L., 2014. Identification of indigenous yeast flora isolated from the five winegrape varieties harvested in Xiangning, China. Antonie Leeuwenhoek 105, 533-540.

Sun, Y., Li, E.H., Qi, X.T. \& Liu, Y.L., 2015. Changes of diversity and population of yeasts during the fermentations by pure and mixed inoculation of Saccharomyces cerevisiae strains. Ann. Microbiol. 2, 911-919.

Torija, M.J., Rozeès, N., Poblet, M., Guillamón, J.M. \& Mas, A., 2001 Yeast population dynamics in spontaneous fermentations: Comparison between two different wine-producing areas over a period of three years. Antonie Leeuwenhoek 79, 345-352.

Tofalo, R., Perpetuini, G., Fasoli, G., Schirone, M., Corsetti, A. \& Suzzi, G., 2014. Biodiversity study of wine yeasts belonging to the "terroir" of Montepulciano d'Abruzzo "Colline Teramane" revealed Saccharomyces cerevisiae strains exhibiting atypical and unique 5.8S-ITS restriction patterns. Food Microbiol. 39, 7-12.

Tofalo, R., Perpetuini, G., Schirone, M., Fasoli, G., Aguzzi, I., Corsetti, A. \& Suzzi, G., 2013. Biogeographical characterization of Saccharomyces cerevisiae wine yeast by molecular methods. Front. Microbiol. 4, 166.

Valero, E., Cambon, B., Schuller, D., Casal, M. \& Dequin, S., 2007. Biodiversity of Saccharomyces yeast strains from grape berries of wineproducing areas using starter commercial yeasts. FEMS Yeast Res. 7, 317 329.

Wang, C.X. \& Liu, Y.L., 2013. Dynamic study of yeast species and Saccharomyces cerevisiae strains during the spontaneous fermentations of Muscat blanc in Jingyang, China. Food Microbiol. 33, 172-177.

White, T.J., Burns, T., Lee, S. \& Tayler, J., 1990. Amplification and direct sequencing of fungal ribosomal RNA genes for phylogenetics. In: Innis, M.A., Gelfand, D.H., Sninsdy, J.J. \& White, T.J. (eds). PCR protocols: A guide to methods and applications. Academics Press, San Diego. pp. 315 -322 .

Zhang, H.Y., Lee, S.A., Bradbury, J.E., Warren, R.N., Sheth, H., Hooks, D.O., Richards, K.D. \& Gardnery, R.C., 2010. Yeasts isolated from New Zealand vineyards and wineries. Aust. J. Grape Wine Res. 16, 491-496. 